\title{
A single latent channel is sufficient for biomedical image segmentation
}

\author{
Andreas M. Kist ${ }^{1, *}$, Anne Schützenberger ${ }^{2}$, Stephan Dürr $^{2}$, Marion Semmler ${ }^{2}$ \\ 1 Department Artificial Intelligence in Biomedical Engineering, \\ Friedrich-Alexander-University Erlangen-Nürnberg, Germany \\ 2 Division of Phoniatrics and Pediatric Audiology at the Department of \\ Otorhinolaryngology, Head and Neck Surgery, University Hospital Erlangen, \\ Friedrich-Alexander-University Erlangen-Nürnberg, Erlangen, Germany \\ * corresponding author: andreas.kist@fau.de
}

\begin{abstract}
Glottis segmentation is a crucial step to quantify endoscopic footage in laryngeal high-speed videoendoscopy. Recent advances in using deep neural networks for glottis segmentation allow a fully automatic workflow. However, exact knowledge of integral parts of these segmentation deep neural networks remains unknown. Here, we show using systematic ablations that a single latent channel as bottleneck layer is sufficient for glottal area segmentation. We further show that the latent space is an abstraction of the glottal area segmentation relying on three spatially defined pixel subtypes. We provide evidence that the latent space is highly correlated with the glottal area waveform, can be encoded with four bits, and decoded using lean decoders while maintaining a high reconstruction accuracy. Our findings suggest that glottis segmentation is a task that can be highly optimized to gain very efficient and clinical applicable deep neural networks. In future, we believe that online deep learning-assisted monitoring is a game changer in laryngeal examinations.
\end{abstract}

\section{Introduction}

A functional voice is a crucial factor for a successful social embedding. Voice physiology is commonly qualitatively assessed using stroboscopy [1]. In contrast, laryngeal high-speed videoendoscopy (HSV) is an emerging technique, that also allows quantification of voice physiology 2 . With HSV, the vocal fold motion is typically recorded at several thousand frames per seconds, therefore visualizing each glottal cycle accurately [3]. The glottis, the opening between the vocal folds, is a good proxy for the cyclic behavior and is of major interest for quantitative data analysis.

The glottis can be segmented using several image analysis techniques [4, among others active contours [5], Gabor filters [6] and thresholding combined with level set methods 7]. Recently, deep neural networks for semantic segmentation have been utilized for glottis segmentation [8,9]. Additionally, optimized deep neural networks for clinical applicability have been proposed [10]. However, these deep neural networks have commonly a black box character, lowering their acceptance in a clinical

environment 11. This effect can be reduced when providing insights to the algorithm. Despite the fact that we know that encoder-decoder architectures and variations thereof are well suited for this task, we are lacking a fundamental understanding of what are the necessities of these deep neural networks. 
Autoencoders or, in general, encoder-decoder architectures have contracting and expansion paths 12,13 , where the bottleneck layer is referred to as code layer or latent space. The latent space is thought to contain a high-level embedding of the raw input image. The inspection of this latent space is highly interesting for generative adversarial networks (GANs), as the latent space can be used in GANs to specifically direct the generative image to enable face editing 14,15], image embedding interpolation [16] and novelty detection [17]. For semantic segmentation, the latent space has also been shown beneficial in multi-task architectures 18,19]. However, little is known what the latent space represents in biomedical image analysis.

In this work, we are characterizing the latent space, a higher order representation of the endoscopic image, embedded in a semantic segmentation architecture. We systematically investigate its properties and how alterations to the latent space result in differences in the glottis segmentation prediction. With this, we will leverage the potential of latent space information in a clinical context.

\section{Materials and methods}

\section{Data and preprocessing}

To train and evaluate deep neural networks, we used the Benchmark for Automatic Glottis Segmentation (BAGLS, 9]). We used the full training and test dataset containing 55,750 and 3,500 images, respectively. For training, we resized all images to $512 \times 256 \mathrm{px}$, which is the native resolution of most images in the dataset. All endoscopic images were converted to grayscale. The input image intensities were normalized to -1 and 1 , the segmentation masks were normalized to 0 and 1 , where 0 is background and 1 the glottal area. We applied data augmentation to the training data randomly using Gaussian blur, rotation ( -30 to $\left.30^{\circ}\right)$, horizontal flip and gamma adjustments. We also use short video snippets of 30 frames available in the BAGLS dataset for time variant data analysis. Videos are processed as single frames on a single frame basis.

\section{Glottal area waveform (GAW)}

The glottal area waveform (GAW) is a one-dimensional representation of the vocal fold oscillation behavior. Each time point of the GAW is computed as the sum of foreground pixels in the glottal area segmentation mask at the given time point [20].

\section{Deep Neural Networks}

\section{Architecture}

The baseline glottis segmentation network is based on the U-Net architecture 21. modified as described in 10. Briefly, we rely on an encoder-decoder architecture to change the image domain from endoscopic image to glottal area segmentation (see Figure 1). Initially, we use skip connections between encoder and decoder to pass mid-level information by concatenation. We setup deep neural networks in TensorFlow 2.6 using the Keras high-level package. We trained for 25 epochs at a constant learning rate of $10^{-4}$ using the Adam optimizer 22. Each convolutional layer used a kernel size of $3 \times 3$ and $f_{L}$ convolutional filters that follow equation 1 .

$$
f_{L}=f_{\text {base }} \cdot 2^{d}
$$

where $f_{L}$ is the number of convolutional filters which equals to the number of channels in a given layer. At a given network depth $d$ (in our baseline model $d \in\{0,1,2,3,4\})$ and a given initial base filter size $f_{\text {base }}\left(f_{\text {base }}=16\right.$ in our baseline 
model) we gain a total of $f_{L}=256$ latent space channels at maximum network depth $d=4$. After each convolution layer, we applied batch normalization. After batch normalization, we used the ReLU function as non-linearity (equation 2). However, in the latent space $\Psi$ (Figure 1A) we used the ReLU6 function (equation 3) that is clipped between 0 and 6 :

$$
\begin{gathered}
\operatorname{ReLU}(x)=\max (0, x) \\
\operatorname{ReLU} 6(x)=\min (\max (0, x), 6) .
\end{gathered}
$$

During training, we were minimizing the Dice loss [23] as defined in equation 4 by comparing the predicted glottis segmentation mask $\hat{y}$ to the ground-truth segmentation mask $y$.

$$
\operatorname{Dice}(y, \hat{y})=1-\frac{2 y \hat{y}+1}{y+\hat{y}+1}
$$

\section{Latent space $\Psi$}

The latent space $\Psi$ is a high-level representation of the initial endoscopy image at the end of the encoder and serves as input to the decoder (Figure 1). It can be interpreted as an image with $f_{L}$ "color" channels. For latent space investigations, we changed $f_{L}$ from its initial value (here: 256), as defined by equation 1 to a fixed value ranging from 1 to $f_{L}$. When $f_{L}=1$, we refer to the latent space as latent space image $\Psi_{1}$.

\section{Decoder experiments}

The initial decoder is constructed as described in the section Architecture. For decoder experiments, we used different strategies to construct the decoder (Figure 5A). The latent space image $\Psi_{1}$ is used as sole input to the decoder. Next, we used a combination of Upsampling2D operations and either one or two convolutional layers with $f_{D}$ channels, where $f_{D} \in\{1,2,4,8\}$. For each Upsampling2D-Convolution cycle $U C \in\{1,2,4\}$, the Upsampling2D operation uses a scaling factor $s \in\{16,8,2\}$, respectively, to ensure a full upsampling to the original image resolution $(512 \times 256 \mathrm{px})$. For training, we converted each training image to its latent space representation by using the final model used in latent space data analysis (result of Figure 1D, with no skip connections and $f_{L}=1$ ). We converted each latent space image in uint8 as we have shown that eight bit are sufficient for high-level encoding (Figure 4 A,B).

\section{Bit encoding}

For evaluation of the bit encoding, we created a histogram of a given latent space image and divided it into $2^{\text {bits }}$ bins. We then set each pixel in a given bin range to the average value in a given bin (Figure $4 \mathrm{C}$ ). The resulting new latent space image is provided to the decoder and the reconstructed image is compared to the ground-truth segmentation mask. We used the mean squared error (MSE) and the intersection over union (IoU) score (see Evaluation) as evaluation metrics.

\section{Evaluation}

We evaluated the segmentation quality using the IoU (intersection over union) score 24 as defined in equation 5 . 


$$
\operatorname{IoU}(y, \hat{y})=\frac{y \cap \hat{y}}{y \cup \hat{y}} .
$$

We further computed the correlation between the latent space image $\Psi_{1}$ (in equation refered as $x$ ) and the $\operatorname{GAW}(y)$ as follows:

$$
\mathrm{r}_{x y}=\frac{\sum_{i=1}^{n}(x-\bar{x})(y-\bar{y})}{(n-1) \cdot s_{x} \cdot s_{y}},
$$

where $n$ is the number of time points/samples, $\bar{x}$ and $\bar{y}$ the average of $x$ and $y$, respectively, and $s_{x}$ and $s_{y}$ the sample standard deviation of $x$ and $y$, respectively.

\section{Code and data availability}

We provide all relevant code at https://github.com/ankilab/latent. In this study, we relied on the open BAGLS dataset. We provide all latent space images for decoder training and the used model for latent space image analysis at

https://zenodo.org/record/5772799.

\section{Results}

\section{A single latent space channel is sufficient for glottis segmentation}

To understand which components are crucial in a segmentation deep neural network, we performed an ablation study on a modified U-Net architecture (see Methods). We trained a full size, complete U-Net to perform glottis segmentation (Figure 1A), similar to previous works 9,10 . The latent space $\Psi$, the ultimate bottleneck that connect encoder and decoder in the full U-Net, has initially 1024 channels (Figure 1A), when 64 filters are used in the first layer $\left(f_{\text {base }}=64\right)$. In this work, we use a reference

implementation with 16 filters in the first layer $\left(f_{\text {base }}=16\right)$ and thus, 256 channels in the latent space, as this has been shown previously to provide comparable performance compared to $f_{L}=1024$ [10]. We systematically reduced the amount of channels in the latent space to determine the minimum viable latent space. We found that even a single latent space channel is sufficient to encode the glottal area segmentation (Figure 1B). However, we hypothesized that the skip connections in the U-Net allow to rescue the strong limitation in the bottleneck. Hence, we removed the skip connections and found that the segmentation accuracy was reduced across configurations (Figure $1 \mathrm{C}$ ). However, the network architecture is still able to provide accurate glottis segmentations (Figure 1D), and has a performance on the test set similar to higher latent space encodings and enabled skip connections. In summary, we show that a single latent space channel is sufficient for glottis segmentation. We will refer to this single latent space channel as latent space image $\Psi_{1}$.

\section{The latent space encodes glottis location and shape}

Next, we investigated the properties of the latent space. We encoded all images of the BAGLS training dataset to gain a collection of latent space $\Psi$ images. We first determined if any pixel is correlated for with the glottal area. We found that the correlation values follow a normal-like distribution centered around 0.00 with a standard deviation of 0.12 (Supplementary Figure ??). We then investigated the value

distribution of the latent space. The histogram shows a distribution centered around 0.8 $($ mean $=0.75$, median $=0.78$, mode $=0.80)$, with a long tail towards 0 and a very short tail 
A

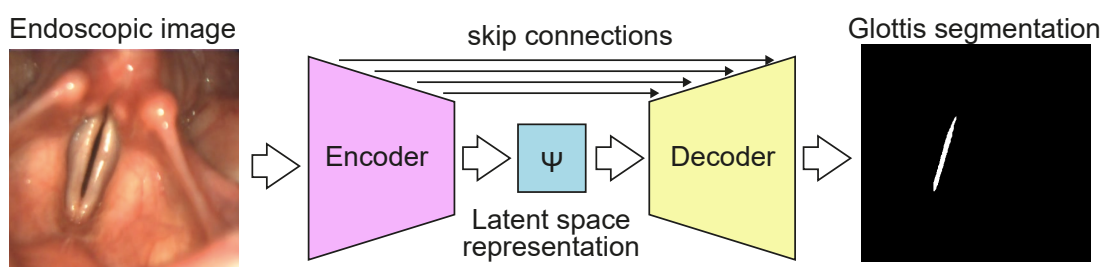

B

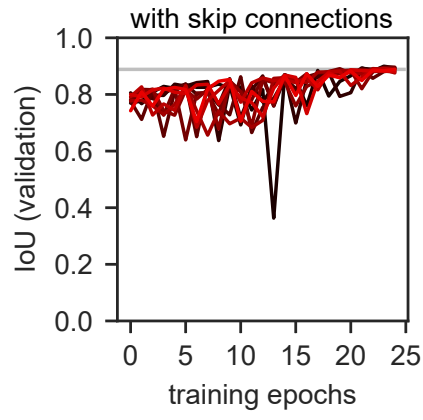

C

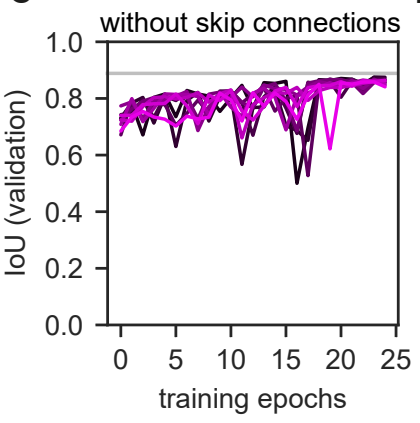

D

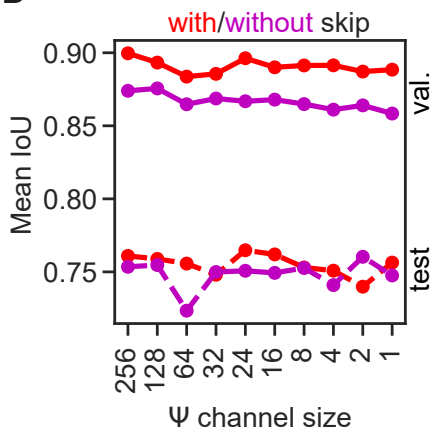

Fig 1. A single latent space channel is sufficient for glottis segmentation. A: Glottis segmentation of endoscopic images using deep neural networks (DNNs) with latent space $\Psi$. B: Convergence of segmentation DNNs across different latent space channels with enabled skip connections. Gradient from black to red indicate less channels. Gray line indicates maximum IoU score. C: Convergence of segmentation DNNs across different latent space channels with disabled skip connections. Gradient from black to magenta indicate less channels. Gray line indicates maximum IoU score from panel B. D: Performance of best performing segmentation DNNs on validation set (solid lines) and evaluated on test set (dashed lines) with enabled (red) and disabled (magenta) skip connections across latent space $(\Psi)$ channels measured by mean intersection over union (IoU).

above 0.8 (Figure $2 \mathrm{~B}$ ). Interestingly, we clipped the available value space in the latent space between 0 and 6 (see Methods), however, the largest value we observed was 1.45 , indicating that we are not constrained by our activation function.

To understand the meaning behind these values, we found that values around 0.8 seem to encode for background (referred to as $\beta$ pixels), values higher than 0.8 define the glottal area (hereafter referred to as $\gamma$ ), and values lower of 0.8 are adjacent to glottal area-encoding values abbreviated $\alpha$ (Figure $2 \mathrm{C}$ ). More examples are shown in Supplementary Figure ??. Further, the latent space image $\Psi_{1}$ is encoding the spatial location of the glottal area in $x$ and $y$. We confirmed this by generating an artificial latent space image $\Psi_{1}$ and varying $x$ and $y$ location, $\gamma$ pixel value intensity and pixel drawing radius (Supplementary Movie 1). Values higher than 1.5 for $\gamma$ resulted in image artefacts. We further investigated the role of the value drop (values $<0.8$ ) for $\alpha$ pixels adjacent to the glottis-defining $\beta$ pixels (values $>0.8$ ). Our results suggest that $\alpha$ pixels in the surrounding of $\gamma$ pixels are shaping the glottal area's extent and refining its appearance (Supplementary Movie 2). Taken together, the interplay between $\alpha$ and $\gamma$ pixels is crucial for an accurate glottis segmentation. 

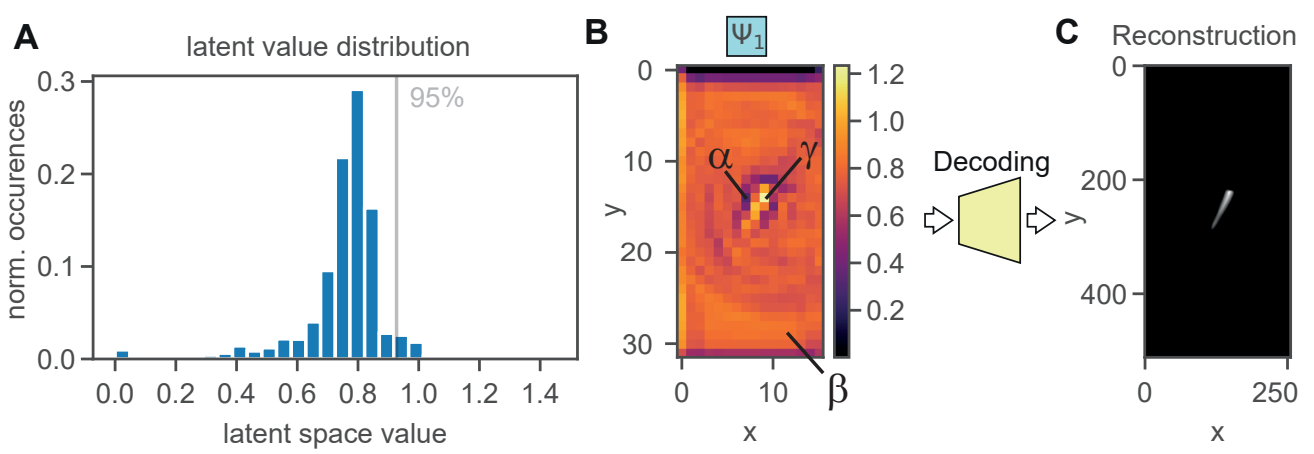

Fig 2. The latent space image $\Psi_{1}$ values provide interpretable context. A: Value distribution of the latent space image $\Psi_{1}$ across all pixels of all images in the BAGLS training dataset. We indicated the $95 \%$ confidence interval that is used for defining $\gamma$ pixels. B: The average latent space image $\Psi_{1}$ across 30 frames. We indicate the three pixel subtypes, $\alpha$ for glottis refining, $\beta$ for background-defining, and $\gamma$ for glottal area defining pixels. C: The average reconstruction obtained from feeding $\Psi_{1}$ from panel B into the decoder.

\section{Thresholded latent space is highly correlated with glottal area waveform}

The glottal area waveform (GAW) is a time-variant signal important for assessing vocal fold physiology 2, 25. We therefore asked, if the latent space image $\Psi_{1}$ is a good proxy for the GAW. To answer this question, we used short video fragments from the BAGLS dataset and converted the provided ground-truth segmentation mask to the GAW (see Methods). We followed two approaches: (1) summing all values in $\Psi_{1}$ and (2) threshold $\Psi$ at $95 \%$ confidence interval (value $=0.8$ ) and then summing the positive pixels. In Figure 3A, we show that approach (1) is correlated to a limited extend with GAWs (on average $0.03 \pm 0.56$ ), however, approach (2) is highly correlated with the GAW, on average $0.84 \pm 0.18$. We show two exemplary videos with corresponding ground-truth GAW, the GAW generated by using the segmentation masks reconstructed by the decoder, and the thresholded $\Psi_{1}$ waveform in Figure $3 \mathrm{~B}$.

\section{A low bit encoding is sufficient for glottis reconstruction}

As the value range is very limited in the latent space (Figure $2 \beta$ ) and the existence of $\alpha$, $\beta$, and $\gamma$ pixels, we hypothesized that a low bit depth is sufficient for encoding the latent space $\Psi_{1}$ for accurate glottal area reconstruction. By reducing the bit depth from 32-bit floating point to a range of 1 to 8-bit, we found that 4-bit encoding is sufficient for high quality reconstructions (Figure 4A-C). Specifically, with 4-bit encoding the IoU score becomes stable and shows a low error, that is neglectable with 8-bit encoding (Figure 4A). The mean-squared error (MSE) between full 32-bit reconstruction and low bit reconstruction declines as expected with increasing bit depth, but is still shows a significant deviation of from the original reconstruction (Figure $4 \mathrm{~B}$ ). We further are able to reproduce the high correlation of $\Psi_{1}$ with the glottal area waveform (Figure $4 \mathrm{D}$ ). In summary, we show that 4-bit encoding is sufficient for subjectively similar glottis segmentations compared to full 32-bit encoding. 
A

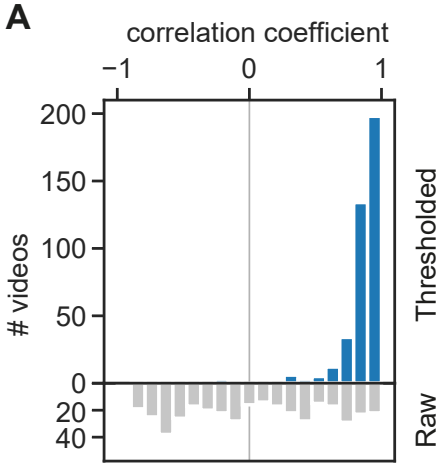

B

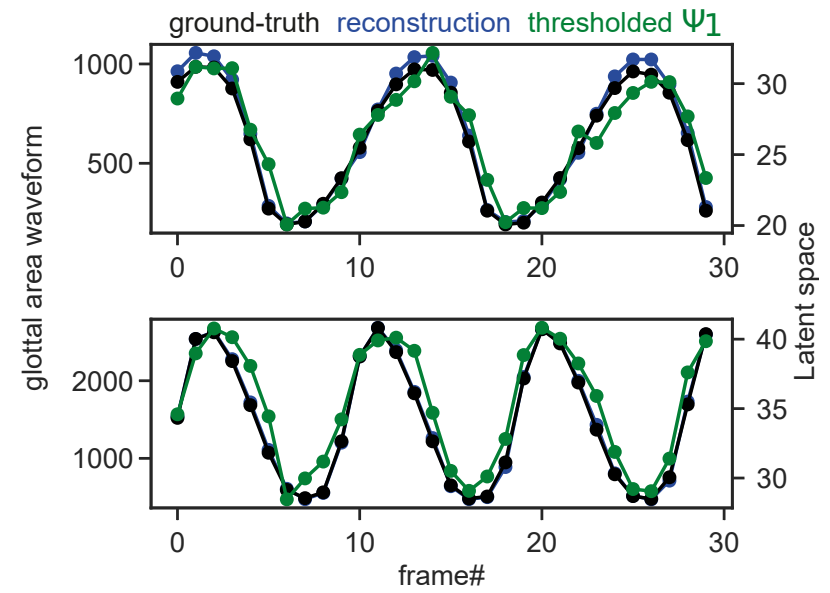

Fig 3. Thresholded latent space image $\Psi_{1}$ is highly correlated with glottal area waveform. A: Distribution of correlation values across videos using either the raw latent space image $\Psi_{1}$ or the thresholded $\Psi_{1}$ using the $95 \%$ confidence interval as threshold (see Figure 2A). Correlation was computed from every latent space to glottal area waveform of 30 frames ( $\mathrm{N}=399$ videos). $\mathrm{B}$ : Two exemplary videos showing the original ground-truth glottal area waveform (GAW, black), the segmentation prediction of the deep neural network with a single latent channel (blue) and the thresholded latent space image $\Psi_{1}$ from the same network (green).

\section{Light-weight decoders are capable of reconstructing the glottal area}

As the latent space image $\Psi$ is easily interpretable and shows a low-level complexity, we hypothesized that the decoder architecture can be largely simplified. Hence, we investigated how many convolutional filters and how many upsampling steps are necessary for decoding (Figure 5A). Further, we were interested if the upsampling strategy (nearest neighbours vs. bilinear interpolation) and multiple convolutional layers are affecting the decoding (Figure $5 \mathrm{~A}$ ). When using a single convolutional layer in each upsampling step, we found that one and two convolutional filters are not sufficient for decoding and that four convolutional filters are only sufficient in a single configuration (4x upsampling and bilinear interpolation) as shown in Figure 5B. The best results were achieved using eight convolutional filters together with 4x upsampling, which resulted in decent IoU scores (0.817, Figure 5B). Using two convolutional layers in each upsampling step, however, allowed $2 \mathrm{x}$ upsampling being competitive in the eight convolutional filters configuration. In general, two and four convolutional filters show better performance compared to the single convolutional layer experiment. However, these are not competitive with the configurations showing eight convolutional filters (Figure $5 \mathrm{C}$ ). The top performance with two convolutional layers per block, eight convolutional filters and $4 \mathrm{x}$ upsampling with $\mathrm{IoU}=0.852$ is slightly outperforming the single convolutional layer configurations. Despite the higher amount of trainable parameters in this configuration (Figure 5D), it has a relatively stable file size of $99 \mathrm{kB}$ (Figure 5E). It is astonishing that even configurations with less than 200 trainable parameters achieve IoU scores higher than 0.4 (Table 1). 
bioRxiv preprint doi: https://doi.org/10.1101/2021.12.10.472122; this version posted December 13, 2021. The copyright holder for this preprint (which was not certified by peer review) is the author/funder, who has granted bioRxiv a license to display the preprint in perpetuity. It is made available under aCC-BY 4.0 International license.

\begin{tabular}{|c|c|c|c|c|c|c|}
\hline Blocks & Filter size & Conv2D layer & Weights & File size $[\mathrm{kB}]$ & IoU (nearest) & IoU (bilinear) \\
\hline \multirow[t]{4}{*}{$1 \mathrm{x}$} & 1 & One & 12 & 19 & 0.01 & 0.01 \\
\hline & 2 & & 24 & 19 & 0.01 & 0.17 \\
\hline & 4 & & 48 & 19 & 0.28 & 0.27 \\
\hline & 8 & & 96 & 19 & 0.42 & 0.29 \\
\hline \multirow[t]{4}{*}{$2 \mathrm{x}$} & 1 & & 23 & 31 & 0.01 & 0.01 \\
\hline & 2 & & 64 & 31 & 0.01 & 0.01 \\
\hline & 4 & & 200 & 31 & 0.60 & 0.45 \\
\hline & 8 & & 688 & 34 & 0.66 & 0.48 \\
\hline \multirow[t]{4}{*}{$4 \mathrm{x}$} & 1 & & 45 & 51 & 0.11 & 0.01 \\
\hline & 2 & & 144 & 51 & 0.40 & 0.48 \\
\hline & 4 & & 504 & 53 & 0.80 & 0.50 \\
\hline & 8 & & 1872 & 58 & 0.82 & 0.79 \\
\hline \multirow[t]{4}{*}{$1 \mathrm{x}$} & 1 & Two & 23 & 30 & 0.01 & 0.01 \\
\hline & 2 & & 64 & 30 & 0.22 & 0.32 \\
\hline & 4 & & 200 & 30 & 0.49 & 0.35 \\
\hline & 8 & & 688 & 33 & 0.39 & 0.35 \\
\hline \multirow[t]{4}{*}{$2 \mathrm{x}$} & 1 & & 45 & 49 & 0.01 & 0.01 \\
\hline & 2 & & 144 & 49 & 0.53 & 0.60 \\
\hline & 4 & & 504 & 51 & 0.46 & 0.68 \\
\hline & 8 & & 1872 & 55 & 0.79 & 0.72 \\
\hline \multirow[t]{4}{*}{$4 \mathrm{x}$} & 1 & & 89 & 84 & 0.01 & 0.01 \\
\hline & 2 & & 304 & 84 & 0.20 & 0.48 \\
\hline & 4 & & 1112 & 88 & 0.71 & 0.61 \\
\hline & 8 & & 4240 & 100 & 0.85 & 0.84 \\
\hline
\end{tabular}

Table 1. Overview of all decoder configurations tested 

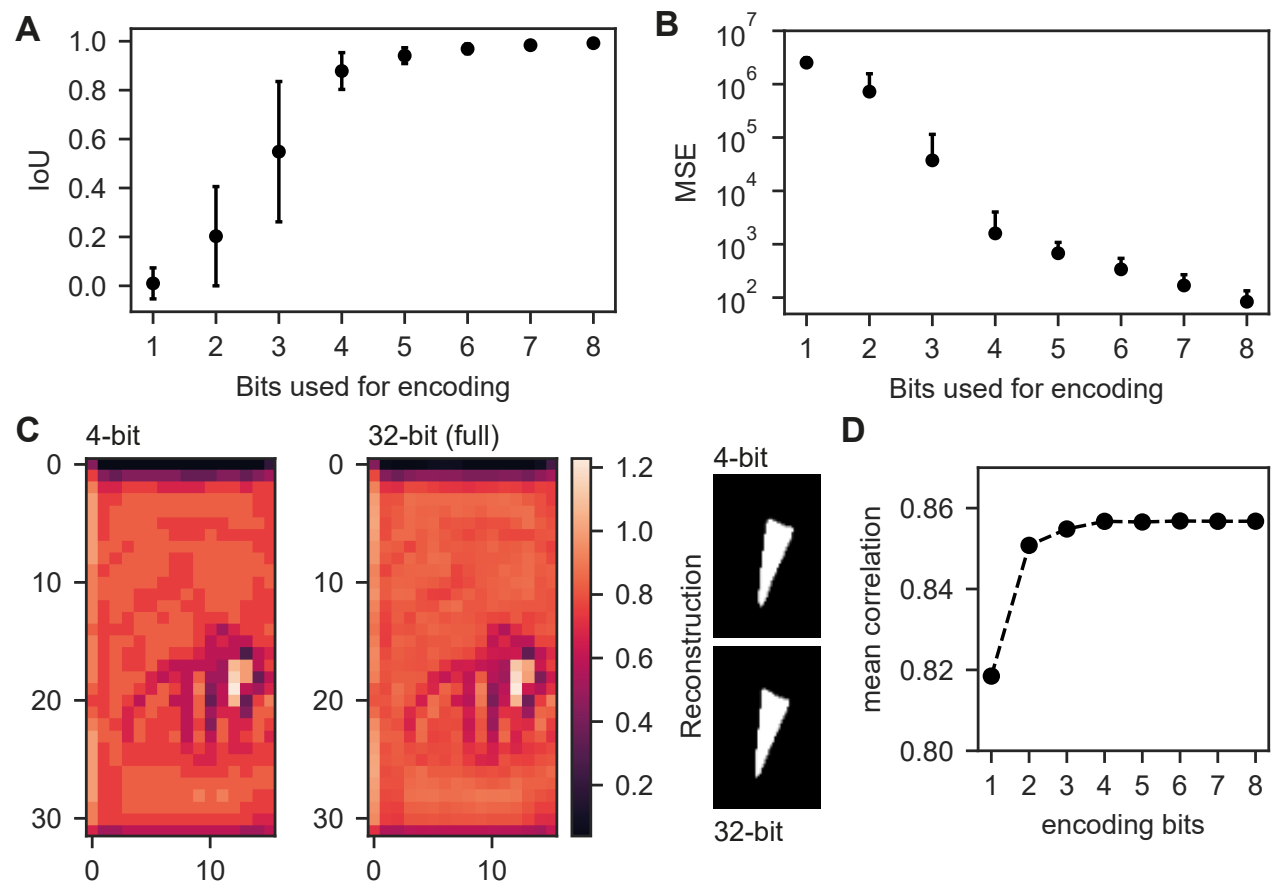

Fig 4. Four bits are sufficient for accurate reconstruction. A: IoU for reconstructions from lower bit encodings and full 32-bit reconstruction. B: Mean squared error (MSE) for lower bit reconstruction and full 32-bit reconstruction. C: Example for 4-bit and respective 32-bit encoding with respective reconstruction. D: Mean correlation of low bit latent space image $\Psi_{1}$ and glottal area waveform (GAW).

\section{Discussion}

In this work, we found that a single channel in the latent space of an encoder-decoder architecture is sufficient for glottal area reconstruction. We further show that the latent space forms an image that has interpretable properties, such as background $(\beta)$, glottal area defining $(\gamma)$ and refining $(\alpha)$ pixels. Our findings suggest that encoder-decoder frameworks are not only suitable for glottis segmentation, but also provide a higher-order approximation of the glottal area sufficiently encoded in a significantly smaller, single channel image. Together with a low bit encoding (Figure 4), it may serve as an efficient data storage system for glottis segmentations. The latent space image can be easily reconstructed using efficient decoders as presented in Figure 5

In this study, we particularily focused on the latent space and its sufficiency for glottis segmentation. We found that removing the U-Net-specific skip connections yielded lower IoU scores in the validation set, whereas we did not find any differences in the test set, i.e. on independent, unseen data (Figure 1D). This is in line with a previous study [10, where the authors showed that the kind of skip connection is not important (adding or concatenating channels from encoder to decoder). They also found a significant drop in the validation IoU score, when removing the skip connections. However, we have not specifically investigated the role of skip connections in this context. It remains elusive if certain data configurations benefit from the skip connections.

Glottis segmentation is a straight forward task and was previously approached using thresholding-based techniques $7,20,26$. Therefore, it is likely that the encoder-decoder architecture learns a non-linear thresholding algorithm. However, other modalities, such 

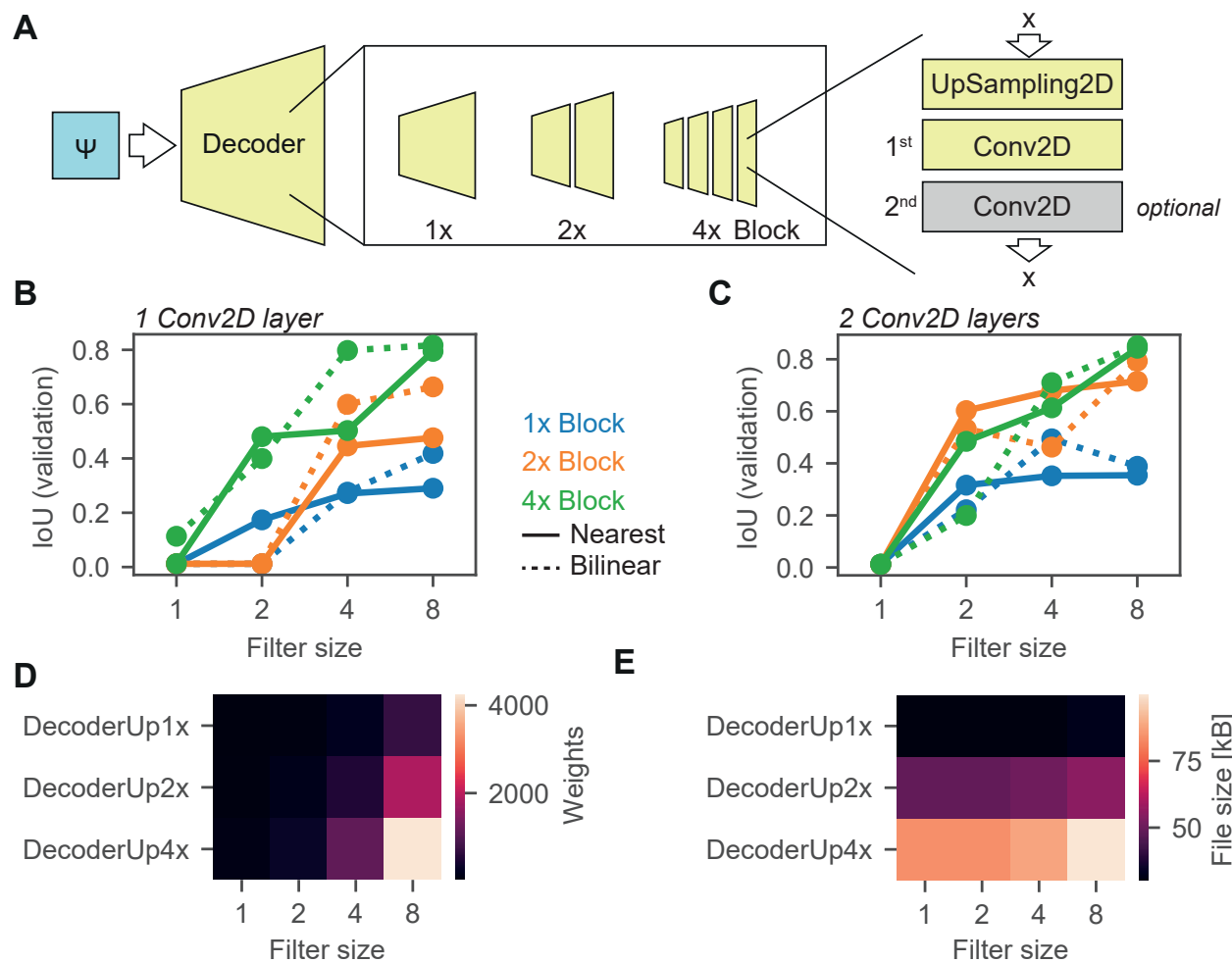

B

D

Filter size
C
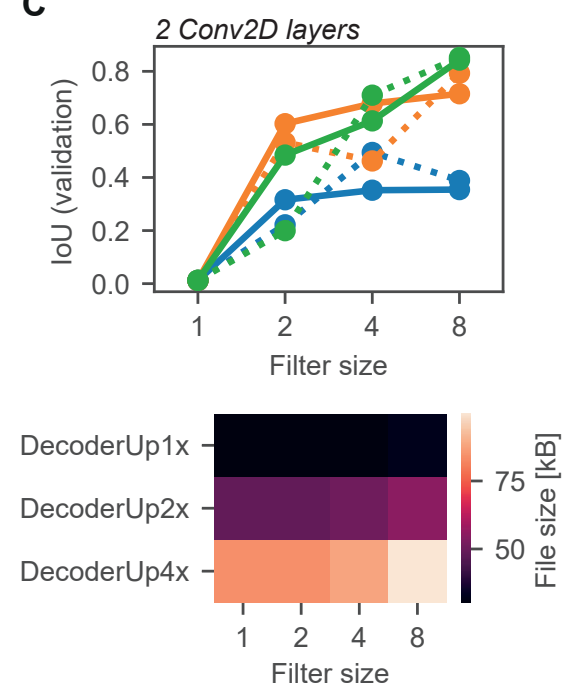

Fig 5. Lean decoders are sufficient for glottis reconstruction. A: Decoding from latent space. The decoder can consist of 1, 2 or 4 upsampling-convolution blocks, wherein one or two convolutional layers can be present. The filter size of each convolutional filter is fixed. B: IoU scores of different decoder blocks (color coded, 1 blue, 2 orange, 4 green) using either nearest neighbour (solid lines) or bilinear upsampling (dashed lines). C: Same as panel B, but with two convolutional layers. D: Trainable weights across decoder settings for two convolutonal layers per block. E: File size across decoder settings for two convolutional layers per block.

as anterior-posterior point prediction for midline estimation 19 and vocal fold localization for paralysis analysis [27] may not benefit from this very constrained latent space. Future studies should address these limitations and speculate about the necessity of an increased latent space crucial for multitask architectures, as the latent space has been shown useful for midline estimation 19 .

The U-Net is a very powerful starting point for biomedical image segmentation tasks, also for glottis segmentation [4,9,28. Modifications to this architecture, such as convolutional layers with LSTM memory cells 29] as shown by 28] may improve the glottal segmentation accuracy. Also, more sophisticated encoding backbones, such as the ResNet [30] and the EfficientNet 31] architecture show superior performance in glottis segmentation, especially on more dissimilar data sources 20]. Future research should investigate, if these architectures are able to detect and encode better high-level features in the latent space, such that a potentially higher dimensionality in the latent spcae yields further performance improvements. 


\section{Conclusion}

253

With this work, we contribute to the understanding how glottis segmentation is performed by deep neural networks and that we are able to uncover the deep neural network black box character by identifying three value ranges with a specific role, namely $\alpha, \beta$ and $\gamma$ pixels. Future studies may elucidate if these three subclasses can be further refined and if they occur across architectures and segmentation tasks. In general, our findings also allow very efficient architectures leveraging the potential of real-time applications of glottis segmentations in a clinical setting and maybe used together with recent advances in open HSV systems 32. Further research on quantitative measures may include how the latent space image $\Psi$ influences these computations and if the latent space is also sufficient for approximating complex quantitative parameters to assess easily voice physiology.

\section{Supporting information}

S1 Fig. Latent space pixels are not directly correlated with glottal area.

S2 Fig. Latent space coding is consistent across recordings.

S1 Video. $\quad \gamma$ pixels define glottal area The glottal area is defined in a range between 0.8 and 1 leading to a nice segmentation. Values higher than 1 tend to create artefacts.

S2 Video. $\alpha$ pixels shape glottal area. This movie shows the interplay between $\alpha$ and $\gamma$ pixels.

\section{Acknowledgments}

This project was supported by Deutsche Forschungsgemeinschaft (DFG) under grant no. SCHU 3441/3-2.

\section{Author contributions}

AMK conceived the project, trained deep neural networks, analyzed the data, created figures and tables. AS, SD and MS interpreted data. AS secured funding. AMK wrote the paper with input from AS, MS and DS.

\section{References}

1. Stachler RJ, Francis DO, Schwartz SR, Damask CC, Digoy GP, Krouse HJ, et al. Clinical practice guideline: hoarseness (dysphonia)(update).

Otolaryngology-Head and Neck Surgery. 2018;158(1_suppl):S1-S42.

2. Deliyski DD, Petrushev PP, Bonilha HS, Gerlach TT, Martin-Harris B, Hillman RE. Clinical implementation of laryngeal high-speed videoendoscopy: challenges and evolution. Folia Phoniatrica et Logopaedica. 2008;60(1):33-44.

3. Kunduk M, Doellinger M, McWhorter AJ, Lohscheller J. Assessment of the variability of vocal fold dynamics within and between recordings with high-speed imaging and by phonovibrogram. The Laryngoscope. 2010;120(5):981-987. 
4. Andrade-Miranda G, Stylianou Y, Deliyski DD, Godino-Llorente JI, Henrich Bernardoni N. Laryngeal image processing of vocal folds motion. Applied Sciences. 2020;10(5):1556.

5. Karakozoglou SZ, Henrich N, d'Alessandro C, Stylianou Y. Automatic glottal segmentation using local-based active contours and application to glottovibrography. Speech Communication. 2012;54(5):641-654.

6. Mendez A, Garcia B, Ruiz I, Iturricha I. Glottal area segmentation without initialization using gabor filters. In: 2008 IEEE International Symposium on Signal Processing and Information Technology. IEEE; 2008. p. 18-22.

7. Gloger O, Lehnert B, Schrade A, Völzke H. Fully automated glottis segmentation in endoscopic videos using local color and shape features of glottal regions. IEEE Transactions on Biomedical Engineering. 2014;62(3):795-806.

8. Laves MH, Bicker J, Kahrs LA, Ortmaier T. A dataset of laryngeal endoscopic images with comparative study on convolution neural network-based semantic segmentation. International journal of computer assisted radiology and surgery. 2019;14(3):483-492.

9. Gómez P, Kist AM, Schlegel P, Berry DA, Chhetri DK, Dürr S, et al. BAGLS, a multihospital benchmark for automatic glottis segmentation. Scientific data. 2020;7(1):186.

10. Kist AM, Döllinger M. Efficient biomedical image segmentation on EdgeTPUs at point of care. IEEE Access. 2020;8:139356-139366.

11. Cadario R, Longoni C, Morewedge CK. Understanding, explaining, and utilizing medical artificial intelligence. Nature Human Behavior. 2021;1.

12. Long J, Shelhamer E, Darrell T. Fully convolutional networks for semantic segmentation. In: Proceedings of the IEEE conference on computer vision and pattern recognition; 2015. p. 3431-3440.

13. Hinton GE, Salakhutdinov RR. Reducing the dimensionality of data with neural networks. Science. 2006;313(5786):504-507.

14. Shen Y, Gu J, Tang X, Zhou B. Interpreting the latent space of gans for semantic face editing. In: Proceedings of the IEEE/CVF Conference on Computer Vision and Pattern Recognition; 2020. p. 9243-9252.

15. Bojanowski P, Joulin A, Lopez-Paz D, Szlam A. Optimizing the latent space of generative networks. arXiv preprint arXiv:170705776. 2017;

16. Abdal R, Qin Y, Wonka P. Image2stylegan: How to embed images into the stylegan latent space? In: Proceedings of the IEEE/CVF International Conference on Computer Vision; 2019. p. 4432-4441.

17. Abati D, Porrello A, Calderara S, Cucchiara R. Latent space autoregression for novelty detection. In: Proceedings of the IEEE/CVF Conference on Computer Vision and Pattern Recognition; 2019. p. 481-490.

18. Saire D, Rivera AR. Empirical Study of Multi-Task Hourglass Model for Semantic Segmentation Task. IEEE Access. 2021;9:80654-80670.

19. Kist AM, Zilker J, Gómez P, Schützenberger A, Döllinger M. Rethinking glottal midline detection. Scientific reports. 2020;10(1):20723. 
20. Kist AM, Gómez P, Dubrovskiy D, Schlegel P, Kunduk M, Echternach M, et al. A Deep Learning Enhanced Novel Software Tool for Laryngeal Dynamics Analysis. Journal of Speech, Language, and Hearing Research. 2021;64(6):1889-1903.

21. Ronneberger O, Fischer P, Brox T. U-net: Convolutional networks for biomedical image segmentation. In: International Conference on Medical image computing and computer-assisted intervention. Springer; 2015. p. 234-241.

22. Kingma DP, Ba J. Adam: A method for stochastic optimization. arXiv preprint arXiv:14126980. 2014;.

23. Milletari F, Navab N, Ahmadi SA. V-Net: Fully Convolutional Neural Networks for Volumetric Medical Image Segmentation. arXiv:160604797 [cs]. 2016;.

24. Jaccard P. Étude comparative de la distribution florale dans une portion des Alpes et des Jura. Bull Soc Vaudoise Sci Nat. 1901;37:547-579.

25. Larsson H, Hertegård S, Lindestad PA, Hammarberg B. Vocal fold vibrations: High-speed imaging, kymography, and acoustic analysis: A preliminary report. The Laryngoscope. 2000;110(12):2117-2122.

26. Lohscheller J, Toy H, Rosanowski F, Eysholdt U, Döllinger M. Clinically evaluated procedure for the reconstruction of vocal fold vibrations from endoscopic digital high-speed videos. Medical image analysis. 2007;11(4):400-413.

27. Adamian N, Naunheim MR, Jowett N. An Open-Source Computer Vision Tool for Automated Vocal Fold Tracking From Videoendoscopy. The Laryngoscope. 2021;131(1):E219-E225.

28. Fehling MK, Grosch F, Schuster ME, Schick B, Lohscheller J. Fully automatic segmentation of glottis and vocal folds in endoscopic laryngeal high-speed videos using a deep convolutional lstm network. Plos one. 2020;15(2):e0227791.

29. Xingjian S, Chen Z, Wang H, Yeung DY, Wong WK, Woo Wc. Convolutional LSTM network: A machine learning approach for precipitation nowcasting. In: Advances in neural information processing systems; 2015. p. 802-810.

30. He K, Zhang X, Ren S, Sun J. Deep residual learning for image recognition. In: Proceedings of the IEEE conference on computer vision and pattern recognition; 2016. p. $770-778$.

31. Tan M, Le Q. Efficientnet: Rethinking model scaling for convolutional neural networks. In: International Conference on Machine Learning. PMLR; 2019. p. 6105-6114.

32. Kist AM, Dürr S, Schützenberger A, Döllinger M. OpenHSV: an open platform for laryngeal high-speed videoendoscopy. Scientific Reports. 2021;11(1):13760. 